\title{
Synthesis of different ZnO nanostructures by modified PVD process and potential use for 1dye-sensitized solar cells
}

\author{
G. Jimenez-Cadena*, E. Comini, M. Ferroni, A. Vomiero, G. Sberveglieri \\ CNR-IDASC SENSOR Lab and University of Brescia, Via Valotti 9, 25133 Brescia, Italy
}

\section{A R T I C L E I N F O}

\section{Article history:}

Received 6 November 2009

Received in revised form 30 March 2010

Accepted 18 July 2010

\section{Keywords:}

PVD

Oxides

Nanostructures

DSSC

\begin{abstract}
A B S T R A C T
Different ZnO nanostructures were synthesized by physical vapor deposition on glass-ITO substrates Nanowires and nanosheets were obtained by a single step process using gold nanoparticles and gold thin films as catalyst. 3D nanoarchitectures were obtained by a two-step modified process; the morphology of these structures depends on the catalyst used for the second deposition: gold nanoparticles or zinc acetate seeds. All the nanostructures were characterized by SEM and TEM analyses, which showed the different morphology under same conditions of temperature, pressure, oxide precursor and deposition time. Dye-sensitized solar cells based on these ZnO structures were successfully assembled, using N179 as sensitizer with efficiencies between $0.1 \%$ and $0.5 \%$. In spite of the low efficiency of the cells, a novel double PVD process is presented and its integration capability into solar cell devices has been proven.
\end{abstract}

(c) 2010 Elsevier B.V. All rights reserved.

\section{Introduction}

Zinc oxide is a semiconductor material with very interesting properties, among them, high sensitivity for gas sensing, piezoelectricity and photoconductivity [1-12]. It has an energy band-gap of $3.37 \mathrm{eV}$ and an exciton binding energy of approximately $60 \mathrm{meV}$, characteristics that make possible its use for different electronic devices as diodes, field-effect transistors, conductometric sensors and dye-sensitized solar cells [11-16,1,8,9]. Nowadays, several techniques are being used to synthesize different nanostructures of this material; sol-gel and hydrothermal methods [7,1,6,4,9,5,17], chemical vapor deposition [18-24] and physical vapor deposition [25,26,2]. The products of these processes include nanoparticles, thin films, nanowires, nanorods and nanobelts.

Physical vapor deposition (PVD) has been used to produce ZnO nanowires networks with a very good repeatability $[11,10,3]$. This process consists of the evaporation of the precursor (metal oxide), the subsequent transport and the final condensation onto the substrate. The growth is catalyzed by metal nanoparticles. It is a relatively simple process and the deposition is controlled by temperature, pressure and carrier gas flow. The nanostructures obtained are tens of nanometers in diameter and several micrometers in length.

The use of different metal oxide nanowires represents a good opportunity to improve the performance in conductometric gas sensors and photovoltaic cells, since their one-dimensional mor-

\footnotetext{
* Corresponding author. Tel.: +39 030 3715767; fax: +39030 2091271

E-mail address: giselle.jimenez@ing.unibs.it (G. Jimenez-Cadena).
}

phology can improve interaction with molecules in gas phase, electronic transport and decrease the electron-hole recombination phenomena. Thavasi et al. [27], have presented the most recent results and the possible improvements using titanium oxide nanorods for dye-sensitized solar cells (DSSC) as well as other effects on the electronic transport on 1D-structures. The use of ZnO one-dimensional for DSSC has been also studied in several works $[28,7,29,30]$ and some improvements in the performance of these devices are expected in the next years.

Other kinds of nanostructures, with different morphologies, can present interesting effects and therefore deserve a proper investigation. In order to produce new $\mathrm{ZnO}$ nanostructured networks, we have introduced some variations to the typical deposition process. These variations include a two steps growth process with second deposition over an already existing nanostructures network. The aim of this methodology is to grow branched structures on the networks of single nanowires.

$\mathrm{ZnO}$ has been quite used for gas sensing [11,10,3] and it is a very promising material for solar cells [14-16]. The exploitation of nanowires in both applications have been investigated by the authors [10,2,3], but in this manuscript we will focus on the results for dye-sensitized solar cells using these new structures.

\section{Experimental \\ 2.1. Physical vapor deposition}

Glass-ITO substrates $\left(10 \mathrm{~mm} \times 10 \mathrm{~mm}, 1.1 \mathrm{~mm}\right.$ of thickness and $20 \pm 5 \Omega \mathrm{sq}^{-1}$ of sheet resistance) were cleaned by acetone, isopropanol and water sonication and then dried with nitrogen. The catalyst, a $10 \mathrm{~nm}$ gold colloidal suspension in agar (BBInternational), was added dropwise onto the substrates and dried at ambient pressure for at least three hours. In order to control the active area 


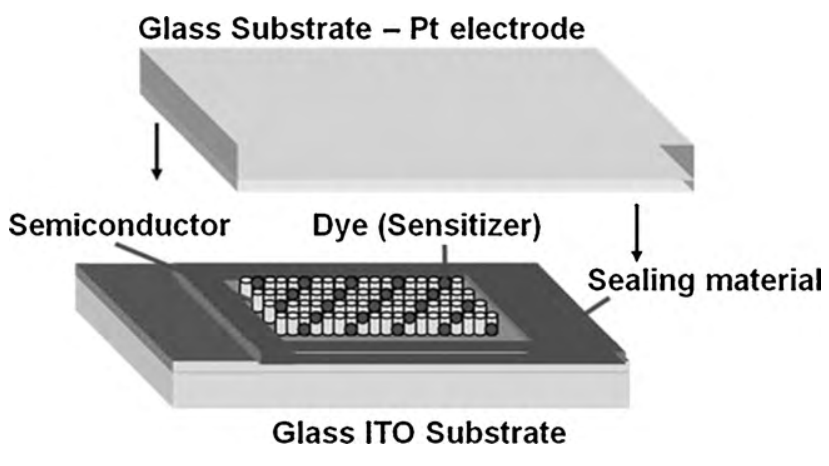

Fig. 1. Dye-sensitized solar cells. of DSSCs, the catalyst was deposited just in the centre of the substrates over a $25 \mathrm{~mm}^{2}$ area. For this, the edges of the substrates were protected using a transparent film before the deposition of the catalyst; the film was removed after drying and before the PVD process. In order to study the influence of the catalyst on the growth, some substrates were prepared using a gold thin film deposited by sputtering. The deposition of gold catalyst was performed by radio frequency sputtering with $50 \mathrm{~W}$ applied power and $7 \times 10^{-3} \mathrm{mbar}$ Ar pressure at room temperature. The deposition was $5 \mathrm{~s}$ long and produced separated clusters with an equivalent thickness in the range of $5 \mathrm{~nm}$. With this technique, a shadow mask can be use to protect the surface of the substrates and to limit the active area for PVD.

The growth of the nanowires was carried out in an alumina tubular reactor at 100 mbar for 20 min using zinc oxide powder (99.999\% Sigma-Aldrich) as precursor. The temperature of the precursor was $1370^{\circ} \mathrm{C}$ while the temperature of the substrates was approximately $350^{\circ} \mathrm{C}$. During the PVD the precursor is transported from the crucible to the substrates with a flux of $75 \mathrm{sccm}$ of argon (carrier gas). In order to control the deposition time and to avoid the transport of the precursor to
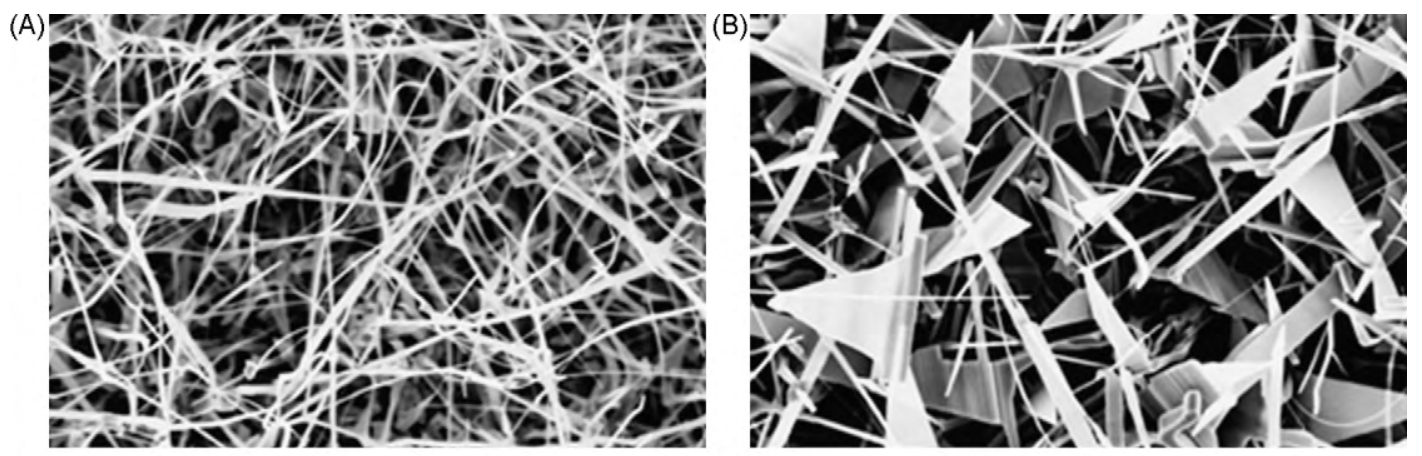

(C)
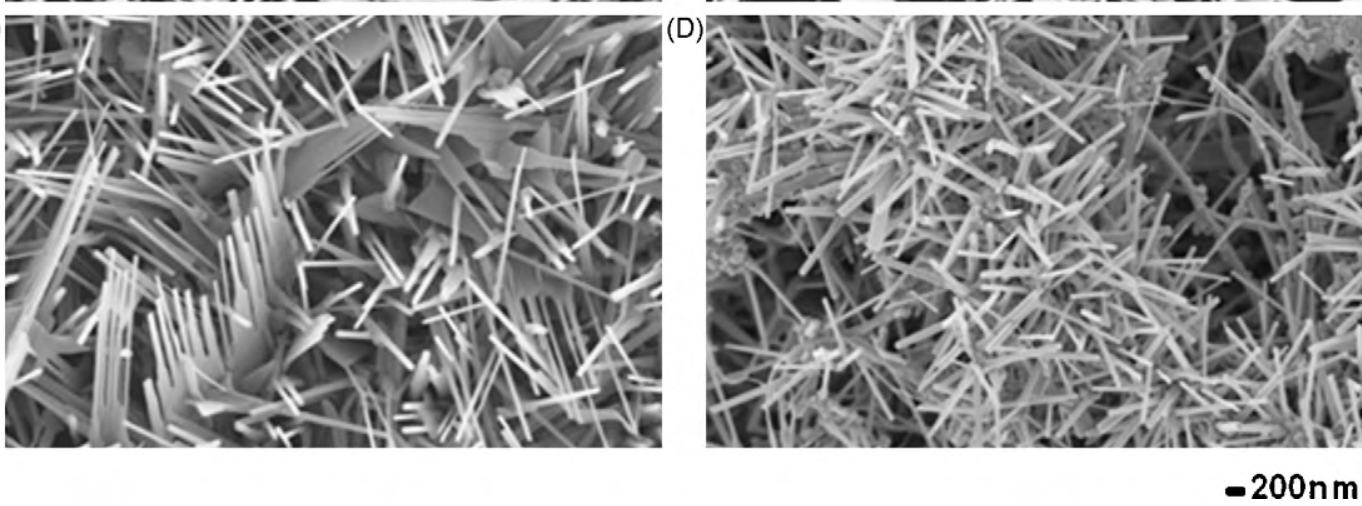

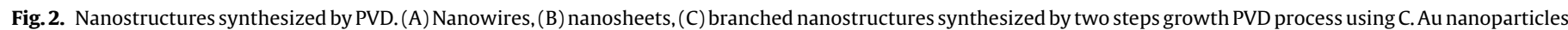
and (D) Zinc acetate seeds as catalyst for the second growth.
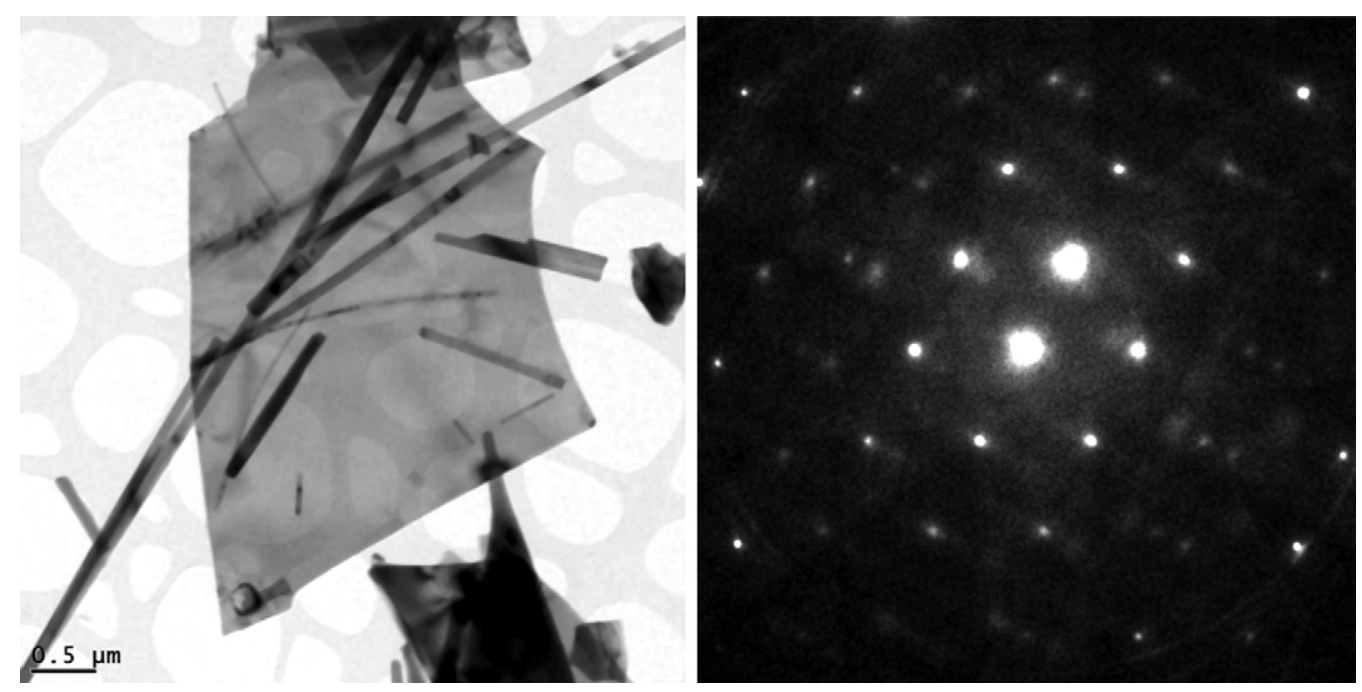

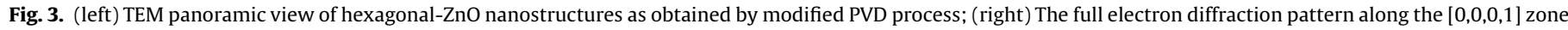
axis from a hexagonal-ZnO nanowire. 
the substrates at lower temperatures, the flux is reversed (from substrates to the crucible) during heating and cooling.

In order to grow branched nanostructures on the $\mathrm{ZnO}$ nanowires, a saturated suspension of zinc acetate dihydrate (99.5\% Fluka) in ethanol was added dropwise onto the substrates with nanowires. At least three drops of this suspension were deposited before the second PVD process; which was done using the same conditions of temperature, pressure and time. A similar process was carried out using Au nanoparticles suspension onto $\mathrm{ZnO}$ nanowires before the second PVD process.

\subsection{Solar cell fabrication}

The organic sensitizer or dye was adsorbed onto $\mathrm{ZnO}$ nanostructures by immersion of the substrates in a ethanol solution of cis-bis(isothiocyanato)bis(2,2' bipyridyl-4,4'-dicarboxylato)ruthenium (II) bis-tetrabutylammonium (dye -N719 solaronix) $0.005 \mathrm{M}$ at room temperature for $16 \mathrm{~h}$. Then, the substrates were washed with ethanol and dried with nitrogen. Glass-ITO substrates coated with a sputtered thin film of Platinum were used as counter electrodes. The two substrates (nanowires-dye and counter electrode) were sealed using a $60 \mu \mathrm{m}$ thick sealing sheet (SX-1170 Solaronix) leaving one of the sides opened where the electrolyte (Iodolyte MPN-100 Solaronix) was injected to fill the space between the two substrates. Finally, the cell was sealed using a wide strip of the sealing sheet. Fig. 1 shows a scheme of the solar cells.

\subsection{Characterization techniques}

SEM images (LEO 1525 FEG-SEM) of the ZnO nanostructures were obtained in secondary electrons imaging mode at an accelerating voltage of $3 \mathrm{kV}$. Transmission electron microscopy (TEM) was carried out with a Tecnai F200 microscope operated at $200 \mathrm{keV}$. The current-voltage curves of the solar cells were obtained using a Solar Simulator Sun 2000 from Abet Technologies under 1.5AM G irradiation condition.

\section{Results and discussion}

\subsection{Nanostructure}

The nanostructures presented in this work contain a network of nanowires, nanosheets and branched structures as it is showed in Fig. 2.

Temperature, pressure, deposition time, precursor and gas carrier flux were similar to synthesize nanowires (1D-structures) and nanosheets (2D structures). However, the size of the catalyst was different for both structures. In order to get a one-dimensional growth, gold thin films or gold nanoparticles $2-5 \mathrm{~nm}$ of diameter; recently deposited; should be use. Films deposited by sputtering are more stable in comparison with the nanoparticles deposited in solution, which tend to aggregate. The agglomeration of the gold nanoparticles produces a bigger size of the catalyst and grows $2 \mathrm{D}$ structures, as the ones showed in Fig. 2B.

The structural properties of $\mathrm{ZnO}$ nanostructures were investigated by TEM, Fig. 3. TEM shows both nanowires and flat platelets of $\mathrm{ZnO}$. The nanostructures have been removed from the substrate through scratching with a sharp blade and released over a conventional holey carbon film grid. The nanowires feature a considerable length-to-width ratio, and a uniform lateral size. The image also shows very small nanowires, preserving their uniform aspect ratio. The smooth dark contrast variations along the nanowires arise from bending of the nanowire, an indication for a high degree of crystallinity. Indeed, the analysis of the electron diffraction pattern reveals a single-crystal assembling for the nanowires. The diffraction pattern, acquired after tilting the nanostructure along a specific crystallographic direction, resulted in an ordered series of systematic rows of sharp spots. The diffraction pattern fits the expected one for hexagonal phase of zinc oxide (Spatial Group 186 with lattice parameters $a=0.325 \mathrm{~nm}$ and $c=0.521 \mathrm{~nm}$, see also pattern 891397 of PCPDF database). The flat structures of $\mathrm{ZnO}$ exhibit the same crystalline assembling of the nanowires. Their different and conventional crystal habits result from the nucleation and growth conditions, which allow formation of large crystalline domains.

For the second PVD just nanowires networks were used. The branched nanostructures obtained by two steps PVD growth, using Au nanoparticles as catalyst for the second deposition process, have

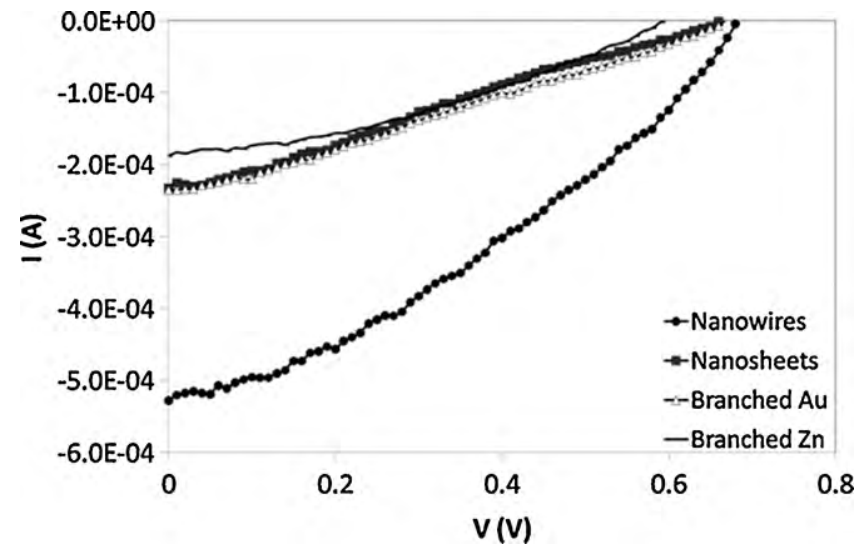

Fig. 4. $I-V$ curves for solar cells based on a network of $\mathrm{ZnO}$ nanowires, $\mathrm{ZnO}$ nanosheets, and $\mathrm{ZnO}$ branched structures obtained by two steps growth PVD using Au nanoparticles and zinc acetate seed as catalyst (Branched Au and Branched $\mathrm{Zn}$, respectively).

showed a one-dimensional growth onto the nanowires during the second PVD. This has favored the formation of a new structure that we have called "nanocombs" as is showed in Fig. 2C. The branched nanostructures grown using zinc acetate seeds as second catalyst had a random growth, forming branched nanostructures as it is showed in Fig. 2D. Differences between both structures suggest a higher concentration of catalyst with zinc acetate seeds, due to the higher density of branches for the second process. This can be produced for a higher affinity of zinc acetate seeds with $\mathrm{ZnO}$ nanowires in comparison with gold nanoparticles. Moreover, if the gold nanoparticles got agglomerated for the second step, a lower concentration of branches would be expected in the double PVD. A deeper study about the synthesis and the growth is necessary to define the mechanism of these processes and conclude about the factor for the different kind of growth.

\subsection{Solar cells}

As possible application for the $\mathrm{ZnO}$ structures, we evaluated their use for DSSC. Figs. 4 and 5 show the $I-V$ and $P-V$ curves for the solar cells based on nanowires, nanosheets and branched nanostructures. The active area of the solar cell is about $5 \mathrm{~mm} \times 5 \mathrm{~mm}$ controlled during the deposition of the catalyst.

The principal parameters of the solar cells (open circuit voltage, short circuit current, fill factor, cell efficiency) are reported in Table 1.

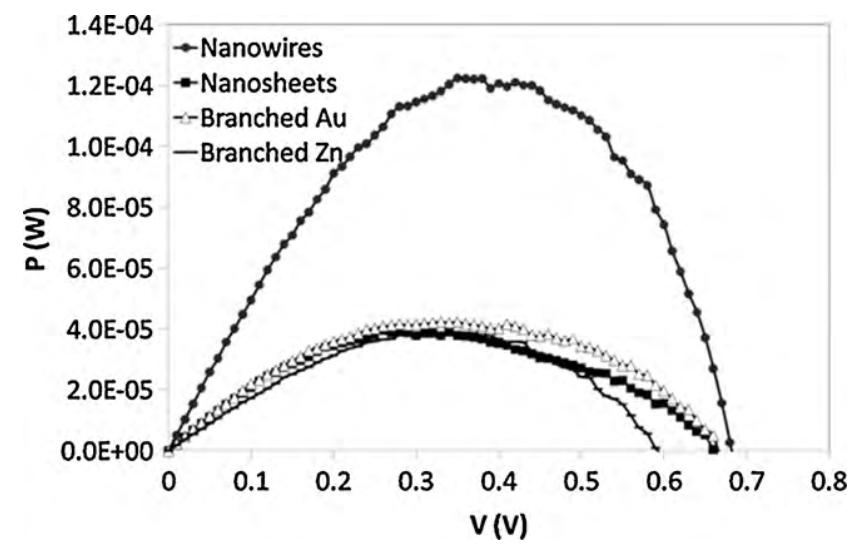

Fig. 5. $P-V$ curves for solar cells based on a network $\mathrm{ZnO}$ nanowires, $\mathrm{ZnO}$ nanosheets, and $\mathrm{ZnO}$ branched structures obtained by two steps growth PVD (Branched Au and Branched Zn). 
Table 1

Open circuit voltage $\left(V_{\mathrm{oc}}\right)$, short circuit current $\left(I_{\mathrm{sc}}\right)$, fill factor $(\mathrm{FF})$ and cell efficiency $(\eta)$ for nanostructures based on $\mathrm{ZnO}$ (Nanowires, nanosheets, branched $\mathrm{Au}$ and branched $\mathrm{Zn}$ ).

\begin{tabular}{lllll}
\hline & $V_{\text {oc }}(\mathrm{V})$ & $I_{\mathrm{sc}}(\mathrm{A})$ & $\mathrm{FF}$ & $\eta$ \\
\hline Nanowires & 0.68 & $-5.28 \mathrm{E}-04$ & 0.34 & 0.51 \\
Nanosheets & 0.66 & $-2.33 \mathrm{E}-04$ & 0.27 & 0.17 \\
Branched Au & 0.66 & $-2.33 \mathrm{E}-04$ & 0.25 & 0.16 \\
Branched $\mathrm{Zn}$ & 0.59 & $-1.88 \mathrm{E}-04$ & 0.35 & 0.16 \\
\hline
\end{tabular}

The $I-V$ and $P-V$ curves show the typical behavior for dyesensitized solar cells. The open circuit voltage values were similar for all the devices as it is expected for different nanostructures of the same composition. However, the short circuit current was clearly higher for the cell based on nanowires. The same difference holds also for the efficiency. For solar cell based on nanowires, $0.51 \%$ of efficiency was reached, while for the other devices, based on nanosheets and branched nanostructures, the efficiency was lower than $0.2 \%$. Based on these results, we can conclude that it is possible to use the $\mathrm{ZnO}$ nanostructures to build dye-sensitized solar cells however; the parameters for the development of the devices should be modified in order to improve the performance of the devices as fill factor and efficiency.

The change in morphology from $1 \mathrm{D}$ to $2 \mathrm{D}$ structures and branched nanostructures can affect the electronic transport on the circuit, since there is not just one-dimensional transport on these new structures. Moreover, the conductivity of glass-ITO substrates is degraded during the heating. The resistance of the substrates can change from $20 \pm 5 \Omega \mathrm{sq}^{-1}$ to $250-300 \Omega \mathrm{sq}^{-1}$. This makes difficult the electronic transport on the circuit and decrease the efficiency of the devices.

As it was expected because of the higher superficial area; adsorption of dye N-179 was higher on substrates with branched nanostructures in comparison with nanowires or nanosheets. However, uniformity on 1D and 2D structures is better since agglomerates of dye molecules are formed on the two steps PVD structures. This can decrease the efficiency of solar cells due to a poor electron injection as it has been explained by Thavasi et al. [27]

Until now it has been supposed that all the nanostructures have the same chemical composition; since they are obtained using the same method, precursor, temperature, pressure and deposition time conditions. However, an analysis of the stoichiometry would be necessary to understand if there are different compositions and the presence of catalyst on the final nanostructured network. Different composition of the nanostructures could modify the semiconductor layer-dye interactions, affecting the dye adsorption and, therefore, the characteristics of the solar cells.

\section{Conclusions}

Several $\mathrm{ZnO}$ nanostructures were synthesized by a repeatable PVD process. 1D and 2D nanostructures were synthesized by a single PVD process and branched nanoarchitectures were grown by a two steps growth PVD using gold nanoparticles and zinc acetate seeds as catalyst for the second.

The conditions for the nanostructures synthesis and the distribution of the catalyst should be studied in order to establish the growth mechanism of the branched nanostructures, which is still under research.
The $\mathrm{ZnO}$ nanostructures synthesized in this work were successfully used to build dye-sensitized solar cells; however, different parameters for the construction of the devices should be optimized to improve the cells characteristics. Also other effects as dye adsorption, electronic transport or composition of the network should be understood to clearly explain the low efficiency of the devices.

\section{References}

[1] P.-C. Kao, S.-Y. Chu, B.-J. Li, J.-W. Chang, H.-H. Huang, Y.-C. Fang, Chang R-C, Low temperature solution-synthesis and photoluminescence properties of $\mathrm{ZnO}$ nanowires, J. Alloys Compd. 467 (2009) 342-346.

[2] G. Sberveglieri, C. Baratto, E. Comini, G. Faglia, M. Ferroni, A. Ponzoni, A. Vomiero, Synthesis and characterization of semiconducting nanowires for gas sensing, Sens. Actuators B: Chem. 121 (2007) 208-213.

[3] E. Comini, C. Baratto, G. Faglia, M. Ferroni, A. Vomiero, G. Sberveglieri, Quasione dimensional metal oxide semiconductors: preparation, characterization and application as chemical sensors, Prog. Mater. Sci. 54 (2009) 1-67.

[4] Z. Li, X. Huang, J. Liu, H. Ai, Single-crystalline $\mathrm{ZnO}$ nanowires on zinc substrate by a simple hydrothermal synthesis method, Mater. Lett. 62 (2008) 2507-2511.

[5] Y. He, W. Sang, J.a. Wang, R. Wu, J. Min, Vertically well-aligned ZnO nanowires generated with self-assembling polymers, Mater. Chem. Phys. 94(2005)29-33.

[6] M.Y. Ge, H.P. Wu, L. Niu, J.F. Liu, S.Y. Chen, P.Y. Shen, Y.W. Zeng, Y.W. Wang, G.Q. Zhang, J.Z. Jiang, Nanostructured ZnO: from monodisperse nanoparticles to nanorods, J. Cryst. Growth 305 (2007) 162-166.

[7] D.I. Suh, S.Y. Lee, T.H. Kim, J.M. Chun, E.K. Suh, O.B. Yang, S.K. Lee, The fabrication and characterization of dye-sensitized solar cells with a branched structure of ZnO nanowires, Chem. Phys. Lett. 442 (2007) 348-353.

[8] A. Khan, W.M. Jadwisienczak, H.J. Lozykowski, M.E. Kordesch, Catalyst-free synthesis and luminescence of aligned ZnO nanorods, Physica E 39 (2007) 258-261.

[9] Q. Zhang, K. Yu, W. Bai, Q. Wang, F. Xu, Z. Zhu, N. Dai, Y. Sun, Synthesis, optical and field emission properties of three different $\mathrm{ZnO}$ nanostructures, Mater. Lett. 61 (2007) 3890-3892.

[10] E. Comini, G. Faglia, G. Sberveglieri, Z.W. Pan, Z.L. Wang, Stable and highly sensitive gas sensors based on semiconducting oxide nanobelts, Appl. Phys. Lett. 81 (2002) 1869-1871.

[11] E. Comini, Metal oxide nano-crystals for gas sensing, Anal. Chim. Acta 568 (2006) 28-40.

[12] Q. Zhang, Y.K.Y. Zhang, Z. Zhu, Photoluminescence field-emission characteristics of $\mathrm{ZnO}$ nanowires synthesized by two-step method, Vacuum 82 (2007) 30-34.

[13] J.K. Song, U. Willer, J.M. Szarko, S.R. Leone, L. Shihong, Y. Zhao, Ultrafast upconversion probing of lasing dynamics in single $\mathrm{ZnO}$ nanowire lasers, J. Phys. Chem. C 112 (2008) 1679-1684.

[14] J.B. Baxter, E.S. Aydil, Dye-sensitized solar cells based on semiconductor morphologies with ZnO nanowires, Sol. Energy Mater. Sol. Cells 90 (2006) 607-622.

[15] B. Pradhan, S.K. Batabyal, A.J. Pal, Vertically aligned $\mathrm{ZnO}$ nanowire arrays in Rose Bengal-based dye-sensitized solar cells, Sol. Energy Mater. Sol. Cells 91 (2007) 769-773.

[16] X. Shen, W. Xu, J. Xu, G. Liang, H. Yang, M. Yao, Quasi-solid-state dye-sensitized solar cells based on gel electrolytes containing different alkali metal iodide salts, Solid State Ionics 179 (2008) 2027-2030.

[17] M. Wang, L. Zhang, The influence of orientation on the photoluminescence behavior of $\mathrm{ZnO}$ thin films obtained by chemical solution deposition, Mater. Lett. 63 (2009) 301-303.

[18] K. Black, A.C. Jones, P.R. Chalker, J.M. Gaskell, R.T. Murray, T.B. Joyce, S.A. Rushworth, MOCVD of $\mathrm{ZnO}$ thin films for potential use as compliant layers for GaN on Si, J. Cryst. Growth 310 (2008) 1010-1014.

[19] L. Dai, H. Deng, G. Chen, J. Chen, Ultraviolet emission properties of ZnO film with zinc deficiency by SS CVD, Appl. Surf. Sci. 254 (2008) 1599-1603.

[20] S. Fay, J. Steinhauser, N. Oliveira, E. Vallat-Sauvain, C. Ballif, Opto-electronic properties of rough LP-CVD ZnO:B for use as TCO in thin-film silicon solar cells, Thin Solid Films 515 (2007) 8558-8561.

[21] Y. Kashiwaba, F. Katahira, K. Haga, T. Sekiguchi, H. Watanabe, Hetero-epitaxial growth of ZnO thin films by atmospheric pressure CVD method, J. Cryst. Growth 221 (2000) 431-434.

[22] Y. Kashiwaba, K. Sugawara, K. Haga, H. Watanabe, B.P. Zhang, Y. Segawa, Characteristics of $c$-axis oriented large grain $\mathrm{ZnO}$ films prepared by low-pressure MO-CVD method, Thin Solid Films 411 (2002) 87-90.

[23] J. Lu, Z. Ye, L. Wang, J. Huang, B. Zhao, Structural, electrical and optical properties of N-doped ZnO films synthesized by SS-CVD, Mater. Sci. Semicond. Proc. 5 (2002) 491-496.

[24] S. Mitra, K. Sridharan, J. Unnam, K. Ghosh, Synthesis of nanometal oxides and nanometals using hot-wire and thermal CVD, Thin Solid Films 516 (2008) 798-802.

[25] Y. Liang, X.T. Zhang, Z. Liu, L. Qin, E. Zhang, C.Z. Zhao, H. Gao, Z.G. Zhang, Local homoepitaxy and optical properties of well-ordered ZnO nanowires, Physica E 33 (2006) 191-195.

[26] Y. Yan, Y. Zhang, G. Meng, L. Zhang, Synthesis of ZnO nanocrystals with novel hierarchical structures via atmosphere pressure physical vapor deposition method, J. Cryst. Growth 294 (2006) 184-190. 
[27] V. Thavasi, V. Renugopalakrishnan, R. Jose, S. Ramakrishna, Controlled electron injection and transport at materials interfaces in dye sensitized solar cells, Mater. Sci. Eng.: R: Rep. 63 (2009) 81-99.

[28] C.H. Ku, J.J. Wu, Electron transport properties in $\mathrm{ZnO}$ nanowire array/nanoparticle composite dye-sensitized solar cells, Appl. Phys. Lett. (2007) 91 .
[29] R. Thitima, C. Patcharee, S. Takashi, Y. Susumu, Efficient electron transfers in ZnO nanorod arrays with N719 dye for hybrid solar cells, Solid-State Electron. 53 (2009) 176-180.

[30] J.J. Wu, G.R. Chen, H.H. Yang, C.H. Ku, J.Y. Lai, Effects of dye adsorption on the electron transport properties in $\mathrm{ZnO}$-nanowire dye-sensitized solar cells, Appl. Phys. Lett. (2007) 90. 